\title{
Defect Analysis of High Electron Mobility Transistors Using a Scanning Electron and Laser Beams Induced Current (SELBIC) System
}

\author{
Haruki Sueyoshi*, Shin-ichi Takasu**, Woon Choi* and Hajime Tomokage* \\ *Department of Electronics Engineering and Computer Science, Fukuoka University, Fukuoka 814-0180, Japan \\ **Advanced Technology Division, JEOL Ltd., Tokyo 196-8558, Japan
}

(Received August 11, 2008; accepted November 25, 2008)

\begin{abstract}
A defect analysis of high electron mobility transistors (HEMTs) was performed using the scanning electron and laser beams induced current (SELBIC) system. An electron gun and two infra red (IR) laser sources with wavelengths of 1064 and $1400[\mathrm{~nm}]$ were located coaxially. Electron and laser beams were irradiated to a GaAs substrate from the reverse side through a quartz window. Two-dimensional current images between gate and source electrodes were observed during the scanning by the 1064 [nm] IR-laser beam under non-biased conditions. The area in which the current changed was observed on the gate electrode, and current leakage was measured from $I-V$ characteristics. The image obtained by simultaneously using two laser beams with wavelengths of 1064 and 1400 [nm] showed different contrasts for each current change.
\end{abstract}

Keywords: Infra Red Laser Beam, Electron Beam, Defect Analysis, HEMT

\section{Introduction}

Defect analysis of electronic devices is vital, because of the industry's (and customers') demands for high reliability, high yield, and short turn around time (TAT). Modern electronic devices are more compact, have higher speed, and achieve higher performance. For miniaturization, IC chips are fabricated with fine patterns and small sized transistors, to produce systems on a chip (SoC). In addition, three-dimensional packages such as stacked die packages and package on packages (PoP) have been applied to advanced electric devices. For defect analysis, therefore, observations must be made of ever-more-complicated structures in ever-smaller areas, making failure detection increasingly difficult.[1] Defects such as voids and Si nodules in ICs and poor interconnection in flip-chip packages are important to discover, because they degrade the performance of the electronic devices and high-speed data transmission.

The electron beam induced current (EBIC) method has been used for defect analysis of IC chips. [2-4] However, in the case of flip chip packages, it is difficult to directly observe the defects of interconnection from the back-side of the IC chip. Therefore, nondestructive observation is needed. The optical beam induced current (OBIC) and optical beam induced resistance change (OBIRCH) methods have been proposed for the failure analysis of semiconductor packages. [5-8] IR (Infrared)-OBIC and IR-OBIRCH methods can be applied to failure analysis from the backside, because IR beams can penetrate most semiconductor substrates.

In this paper, we report the defect analysis of high electron mobility transistors (HEMTs) using a scanning electron and laser beams induced current (SELBIC) system. The induced currents of HEMTs were observed in a vacuum with electron and IR laser beam irradiation from the backside under non-biased conditions. First, an IR-beam with a wavelength of 1064 [nm] irradiated the HEMT, and then an electron beam irradiated the various spots under the IR-beam scanning. Finally, two IR-beams (wavelengths of 1064 and 1400 [nm]) were used simultaneously to irradiate the HEMTs.

\section{Experimental Procedure}

Figure 1 shows the SELBIC system, which consists of main console, control rack, and operation console. A cold cathode field emission gun and laser sources with wave- 


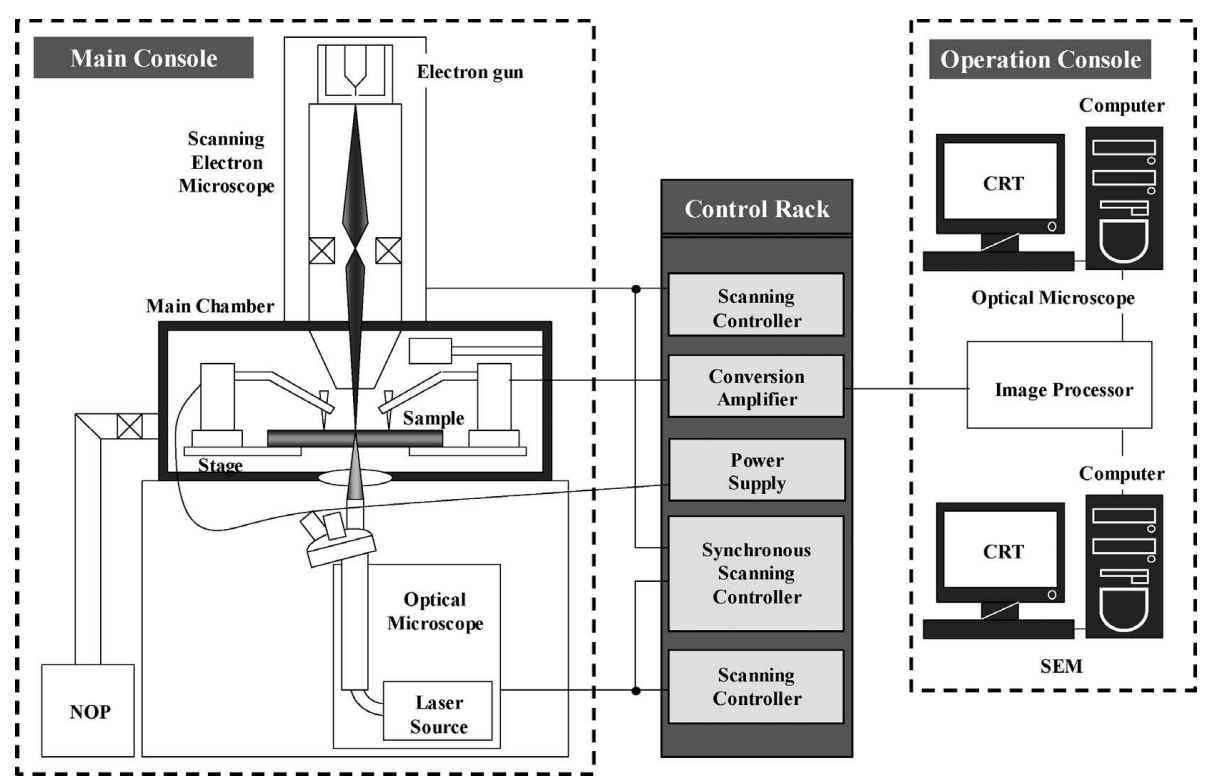

Fig. 1 Schematic diagram of SELBIC system.

lengths of 1064 [nm] (YAG laser) and 1400 [nm] (laser diode) are located coaxially across the vacuum chamber. The laser beams irradiate the specimen through a quartz window. The specimen can be observed under non-bias or bias conditions in still-air or in a vacuum $\left(9.63 \times 10^{-5}[\mathrm{~Pa}]\right)$. The specimen is connected to tungsten probes in order to observe the induced current. There are three scanning controllers for the electron beam and the laser beams. In the SELBIC system, scanning electron microscopy (SEM) and optical observation are performed simultaneously using each scanning controller. In addition, the electron beam and laser beam irradiate the same area at the same time using a synchronous scanning controller. The two beams can be also guided to irradiate any spot. The induced current is amplified by the conversion amplifier. The output signal is synchronized with the scanning signal, so that the two-dimensional current image can be displayed on the CRT.

Table 1 compares the SELBIC method with conventional defect observation methods that use electron or laser beams. The basic functions of the SELBIC system are optical laser microscopy (OM) and scanning electron microscopy (SEM). An optical beam induced current (OBIC) technique and an optical beam induced resistance change (OBIRCH) technique have been proposed as ways to apply $\mathrm{OM}$ for defect characterization. In particular, observation under non-biased conditions is called the NB (non-bias)-OBIC method.[9] On the other hand, SEM is used as an electron beam induced current (EBIC) method for detecting failures in the depletion layer. In both OBIC
Table 1 Comparison with SELBIC and conventional observation.
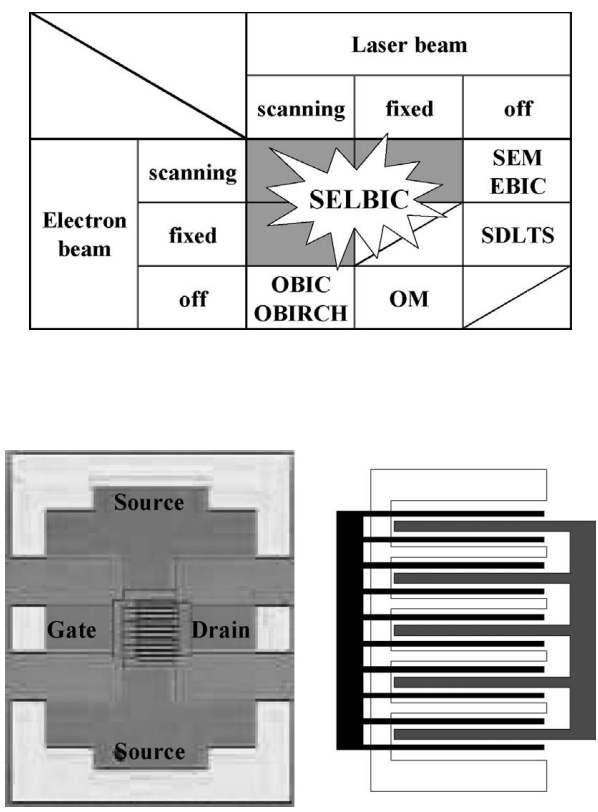

Fig. 2 HEMT and the comb-like structure.

and EBIC measurements, the current induced by the optical beam or electron beam is measured as the current image. The laser beam is used for the heat source in the OBIRCH method. The SELBIC method is unique in that it uses the electron beam and laser beam simultaneously in the same area. The beams can be either scanning or fixed while observing the current change.

Figure 2 shows a HEMT with a comb-like structure on a GaAs substrate. The HEMT has a gate, a drain, and source electrodes. Tungsten probes are connected to the 
gate and source electrodes in the vacuum chamber. In the vacuum, the induced currents of HEMTs with different numbers of gates were observed from the backside under non-biased conditions. First, an IR-beam with a wavelength of 1064 [nm] irradiated the HEMT, and then an electron beam irradiated various spots under the scanning IR-beam. In addition, observations were made of the induced current using IR-laser beams with wavelengths of 1064 and $1400[\mathrm{~nm}]$.

\section{Results and Discussion}

Figures 3 and 4 show the optical image (a) and NBOBIC image (b) of HEMT samples \#1 and \#2, respectively. The 1064 [nm] IR-laser beam irradiated the HEMT from the backside in a vacuum. Optical images of the HEMT with two gate electrodes were clearly observed. The current change along the gate electrode could be observed in the NB-OBIC image of sample \#1, shown in Fig. 3(b). On the other hands, a large area of current change was observed in the NB-OBIC image of sample \#2, shown in Fig. 4(b). This current changed area could be considered a leakage spot on the gate electrode.

Figure 5 shows the SEM image of sample \#2. With the SELBIC system, OBIC and SEM observations were performed simultaneously. By comparing the images we can see that the large current changed area was not the result

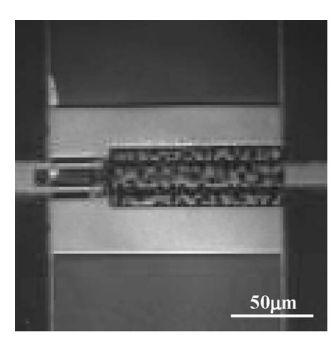

(a) Optical image

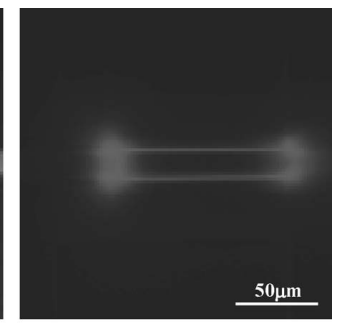

(b) NB-OBIC image
Fig. 3 Optical image (a) and NB-OBIC image (b) of sample \#1.

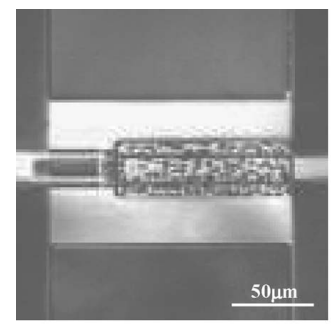

(a) Optical image

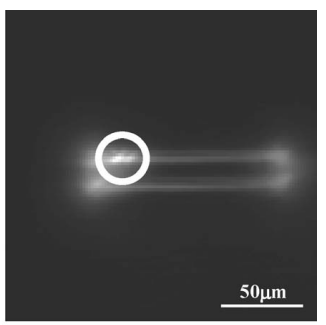

(b) NB-OBIC image
Fig. 4 Optical image (a) and NB-OBIC image (b) of sample \#2. of surface damage, because there was no area corresponding to it in the SEM image.

Figure 6 shows the $I-V$ characteristics of samples \#1 and \#2. Voltage from 0 to 550 [mV] was applied between the gate and source electrode. Sample \#1 shows good diode characteristics, but leakage current was measured from sample \#2, shown in Fig. 6.

Figure 7 compares the NB-OBIC and SELBIC images. A tungsten probe was connected to the gate and source electrodes, and then a YAG laser beam with power of $4[\mathrm{~mW}]$ was used to irradiate the HEMT. After the NB-OBIC observations, an electron beam of accelerating voltage 25 $[\mathrm{kV}]$ irradiated the area around the gate electrode. Figure

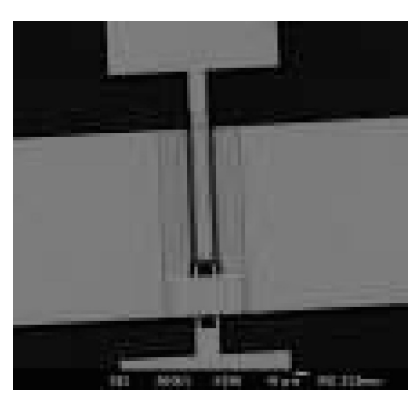

Fig. 5 SEM observation.

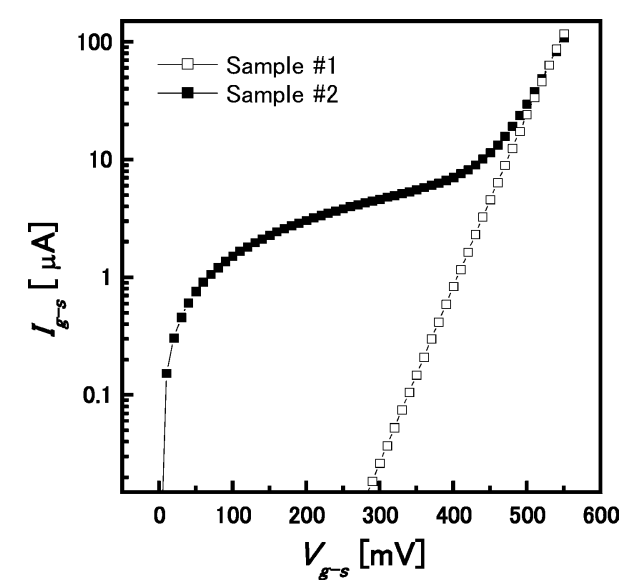

Fig. $6 \quad I-V$ characteristics of samples \#1 and \#2.

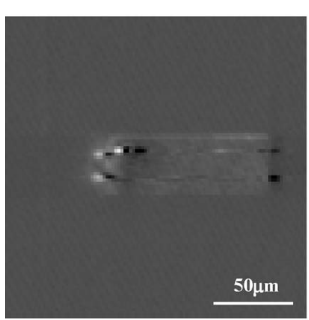

(a) NB-OBIC image

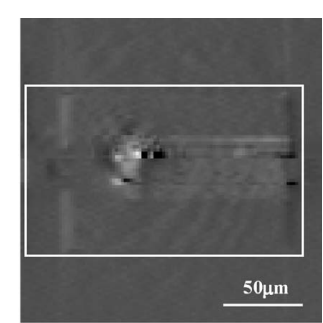

(b) SELBIC image
Fig. 7 NB-OBIC image (a) and SELBIC image (b) irradiated electron beam of the accelerating voltage $25 \mathrm{kV}$. 


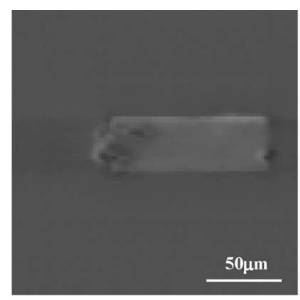

(a)YAG: $1 \mathrm{~mW}$

$\mathrm{LD}: 3 \mathrm{~mW}$

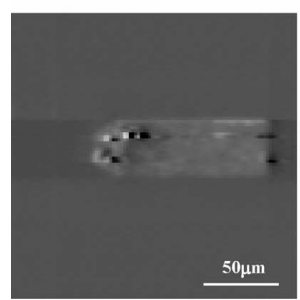

(c)YAG: $14 \mathrm{~mW}$

$\mathrm{LD}: 3 \mathrm{~mW}$

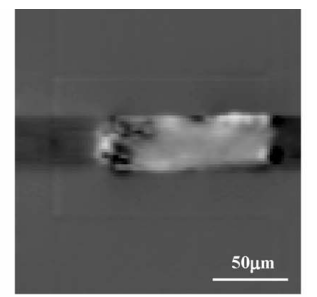

(b)YAG: $1 \mathrm{~mW}$

LD: $17 \mathrm{~mW}$

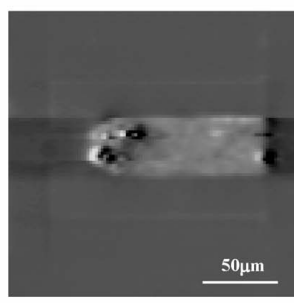

(d)YAG: $14 \mathrm{~mW}$

LD: $17 \mathrm{~mW}$
Fig. 8 OBIC images with wavelength of 1064 and 1400 [nm].

7(b) shows the SELBIC image. A new contrast appeared around the irradiation point from the electron beam irradiation. New contrast around the irradiation point is the electrode shape that could not appear in the NB-OBIC image, and considered that the injected electrons lead the current change.

Figure 8 shows the OBIC images using a YAG laser and laser diode at various laser powers: (a)YAG:1 [mW], LD:3 [mW]; (b)YAG:1 [mW], LD:17 [mW]; (c)YAG:14 [mW], LD:3 [mW]; and (d)YAG:14 [mW], LD:17 [mW]. We refer to the OBIC image obtained with two laser beams as the T(twin)-OBIC image. We can see that T-OBIC shows a contrast different from that in the NB-OBIC image; the brighter contrast in the T-OBIC image is a result of the higher laser power. On the other hands, the beam of the laser diode heats the irradiation point. Therefore, in the case of high YAG laser power, the current change depends on the induced current. However, in the case of high laser diode power, the current change is induced by local heat- ing.

\section{Conclusion}

SELBIC measurements were made of HEMTs on GaAs substrates using laser beams with two different wavelengths and an electron beam. A comparison of the $I-V$ characteristics between gate and source electrodes suggested that the high contrast of the obtained NB-OBIC image was due to gate leakage. Simultaneous irradiation by a laser beam and an electron beam created a new contrast in the NB-OBIC image. In addition, we found the OBIC image made using two laser beams was affected by the balance of laser power. These results, indicate that SELBIC method is a powerful tool for the defect analysis of semiconductor devices.

\section{References}

[1] C. E. Bauer and H. J. Neuhaus, Proc. ICEP 2007, pp. 27-32 (2007).

[2] H. Tomokage, Y. Ishiwata, H. Souno, M. Kawakami, and N. Sonoda, Solid-State Phenomena, 63-64, pp. 407-412 (1998).

[3] V. K. S. Ong, P. C. Liu and K. T. Lau, Solid-State Electronics, 43, pp. 41-50 (1999).

[4] V. K. S. Ong and D. Wu, Solid-State Electronics, 44, pp. 1585-1590 (2000).

[5] E. Ramsay, D. T. Reid and K. Wilsher, Appl. Phys. Lett., 81, pp. 7-9 (2002).

[6] H. Komoda and K. Shimizu, Jpn. J. Appl. Phys., 33, pp. 3393-3401 (1994).

[7] K. Nikawa, C. Matsumoto and S. Inoue, Jpn. J. Appl. Phys., 34, pp. 2260-2265 (1995).

[8] K. Nikawa and S. Inoue, Proc. DRIP VII, Inst. Phys. Conf. Series No. 160. Inst. Physics Pub., Bristol and Philadelphia, pp. 37-46 (1998).

[9] T. Koyama, K. Sonoda, J. Komori and Y. Mashiko, J. Appl. Phys., 86, pp. 5949-5956 (1999). 